
One Brookings Dr, St. Louis, MO, 63130

drewjmclaughlin@wustl.edu kvanengen@wustl.edu

24 
PUPIL RESPONSE FOR ACCENTED SPEECH

Abstract

2 Unfamiliar second-language (L2) accents present a common challenge to speech understanding.

3 However, the extent to which accurately-recognized unfamiliar L2-accented speech imposes a

4 greater cognitive load than native speech remains unclear. The current study used pupillometry

5 to assess cognitive load for native English listeners during the perception of intelligible

6 Mandarin Chinese-accented English and American-accented English. Results showed greater

7 pupil response (indicating greater cognitive load) for the unfamiliar L2-accented speech. These

8 findings indicate that the mismatches between unfamiliar L2-accented speech and native

9 listeners' linguistic representations impose greater cognitive load even when recognition

10 accuracy is at ceiling.

11

12 Keywords: speech perception; accent; pupillometry 


\section{PUPIL RESPONSE FOR ACCENTED SPEECH}

\section{I. INTRODUCTION}

2 Listening to unfamiliar second language- (L2-) accented speech is often described by listeners as

3 an effortful process, even when L2 speakers are highly proficient (Munro \& Derwing, 1995).

4 Both L2- and regionally-accented speech are characterized by systematic segmental and

5 suprasegmental deviations from standard native pronunciations of a given language or dialect.

6 For example, when produced by nonnative Mandarin Chinese speakers of English, tense (e.g.,

7 /i/) and lax (e.g., /I/) American English vowel pairs tend to be less phonetically distinct than

8 when produced by native speakers (Wang \& Heuven, 2006). Deviations such as these result in

9 mismatches between L2 speech and native listeners' representations, which may cause reduced

10 recognition accuracy (the proportion of words in the speech stream that can be correctly

11 identified) and/or increased cognitive load (the degree to which cognitive resources are recruited

12 at a given moment to meet processing demands; Pichora-Fuller et al., 2016). That is, resolving

13 such mismatches may require additional cognitive resources not needed for native-accented

14 speech (see Van Engen \& Peelle, 2014, for an executive recruitment account of accented

15 speech). Furthermore, even when unfamiliar L2-accented speech is accurately recognized,

16 processing it should nonetheless require greater cognitive load than processing native speech.

17 Our research question was the following: Does unfamiliar L2-accented speech that is

18 accurately recognized impose greater cognitive load than native speech? Behavioral studies have

19 primarily relied on performance measures such as recognition accuracy (also referred to as

20 "intelligibility") and reaction time (e.g., processing speed for true vs. false sentence verification;

21 Adank, Evans, Stuart-Smith, \& Scott, 2009) to determine the costs of processing unfamiliar L2-

22 accented speech. However, examining the cognitive load associated with the online processing of

23 speech from highly proficient L2 speakers, including those whose speech can be accurately 


\section{PUPIL RESPONSE FOR ACCENTED SPEECH}

1 recognized, requires a different methodological approach. To address our research question, we

2 used pupillometry (the measure of pupil diameter over time) to examine cognitive load for native

3 English listeners during the perception of accurately-recognized Mandarin Chinese-accented

4 English and standard American-accented English. By using pupillometry in place of recognition

5 accuracy and/or reaction time measures, we were able to examine the time-course of effortful

6 processing for accurately-recognized speech. Notably, our definition of "recognition accuracy" is

7 the proportion of keywords that the participant is able to correctly repeat, and does not

8 necessarily reflect what the participant initially perceived; in fact, our hypothesis is predicated on

9 the assumption that listeners are recruiting additional cognitive resources to re-process their

10 initial perceptions. Further discussion of the mechanism(s) hypothesized to support this process

11 are revisited in the discussion.

12 Pupillometry has been used for several decades as a non-intrusive, temporally-sensitive

13 psychophysiological index of cognitive load (Beatty, 1982), and more recently has been applied

14 within the domain of speech perception (for a review, see Van Engen \& McLaughlin, 2018). Of

15 interest for cognitive research is the task-evoked pupil response (from here forward simply pupil

16 response), which is a phasic increase of pupil diameter linked temporally to a specific cognitive

17 event (Beatty, 1982). These increases in pupil diameter are much smaller than changes due to

18 luminance, and are related to up-regulation of sympathetic, and inhibition of parasympathetic,

19 activity (Steinhauer, Siegle, Condray, \& Pless, 2004; Beatty \& Lucero-Wagoner, 2000;

20 Peysakhovich, Vachon, \& Dehais, 2017). Larger pupil response during a cognitive task indicates

21 greater cognitive load. For example, Beatty (1982) found that pupil response was systematically

22 larger for longer digit spans during a recall task. 


\section{PUPIL RESPONSE FOR ACCENTED SPEECH}

Using acoustically degraded speech, it has been demonstrated that the pupil response increases systematically as speech becomes less intelligible (Zekveld, Kramer, \& Festen, 2010;

3 Zekveld \& Kramer, 2014). Additionally, for Dutch-English bilingual listeners, pupil response for

$450 \%$ intelligible English (i.e., L2) speech is greater when presented in English babble than in

5 Dutch babble, and is greater when the speaker's language is not known in advance (i.e., when

6 trials are randomized as opposed to blocked; Francis, Tigchelaar, Zhang, \& Zekveld, 2018).

7 Porretta and Tucker (2019) examined the effect of intelligibility using L2-accented speech,

8 demonstrating for isolated words that there is greater and more sustained pupil response for

9 unintelligible accented speech than intelligible accented speech. However, the potential

10 difference in pupil response for accurately recognized, unfamiliar L2-accented speech and native

11 speech remains to be investigated.

12 In a study using noise-vocoded speech, Winn, Edwards, and Litovsky (2015) found that

13 even when analyses were limited to accurately-perceived trials, there was a systematic increase

14 in pupil response as the spectral quality of the speech signal decreased. Thus, pupillometry is

15 capable of capturing cognitive load as a function of recognition accuracy, but also revealing

16 more nuanced differences in cognitive load when recognition is at ceiling. The latter makes

17 pupillometry a valuable method for addressing our research question, and offers a novel way to

18 quantify the challenge posed by accented speech.

In the present study, we measured pupil response during listening to speech from a

20 native, standard American-accented speaker of English and a nonnative, Mandarin Chinese-

21 accented speaker of English. We predicted that growth curve analysis (Mirman, 2014) of the

22 pupil response would show an effect of speaker type (i.e., overall pupil diameter would be

23 greater for the L2-accented speaker condition), and an interaction between speaker type and the 


\section{PUPIL RESPONSE FOR ACCENTED SPEECH}

1 linear time term (i.e., indicating a difference in the rate of change of pupil diameter between

2 conditions), reflecting greater cognitive load for processing L2-accented speech than native

3 speech. Additionally, we predicted that subjective ratings of listening effort would be greater for

4 the L2-accented speaker condition. Study preregistration is available at: https://osf.io/bgcx 7/; all

5 analysis scripts and data are available at: https://osf.io/7dajv/files/.

\section{II. METHODS}

\section{1. Participants}

8 Participants were young adults $(N=52$, ages 18-22, 39 female and 13 male) recruited from

9 Washington University's Psychology Subjects Pool. Hearing thresholds were measured for each

10 participant to confirm they had clinically normal hearing. An additional 11 subjects participated

11 and were excluded and replaced due to data loss either from equipment malfunction, or because

12 they were not native speakers of American English, had parents who were not native speakers of

13 American English, or had extensive exposure to Mandarin Chinese.

\section{2. Stimuli}

15 Sentence recordings of a native speaker of standard American English and a Mandarin-Chinese

16 accented speaker of English were taken from Van Engen, Chandrasekaran, and Smiljanic (2012).

17 All sentences were six words long, with four keywords (e.g., "the gray mouse ate the cheese").

18 Results from a pilot study (see Supplemental Materials) indicated that the Mandarin Chinese-

19 accented stimuli had high recognition accuracy but were also rated as more accented and more

20 effortful to understand than the native-accented stimuli. Root mean squared amplitudes for all

21 stimuli were equalized using Praat (Boersma \& Weenink, 2019). To control for natural

22 differences in speaking rate, which is crucial for interpreting the time-course of the pupil

23 response, the native speaker files were acoustically lengthened. Adjustments were made using 


\section{PUPIL RESPONSE FOR ACCENTED SPEECH}

1 the Stretch function in Adobe Audition CC, which can adjust file lengths while controlling for

2 pitch and speech characteristics. Files were lengthened by a constant amount, so that the average

3 length of the native speaker files would equal the average length of the L2 speaker files. The L2

4 speaker files were not altered. A pilot experiment with unprocessed native stimuli is reported in

5 the Supplemental Materials. The same pattern of results was found.

\section{3. Procedure}

7 Participants provided written informed consent prior to participation in the study. Following the

8 consent process, they completed language and demographic questionnaires and underwent

9 audiometric testing. Puretone thresholds at $500 \mathrm{~Hz}, 1000 \mathrm{~Hz}$, and $2000 \mathrm{~Hz}$ were used to establish a

10 pure-tone average (PTA) for each participant. All participants had PTAs at or below 10dB in at

11 least one ear.

12 For the pupillometry task, participants were seated in a sound-attenuating room facing a

13 monitor and EyeLink 1000 Plus camera. All equipment was positioned following EyeLink

14 specifications. During the experiment, participants rested their chins on a head-mount. They

15 were instructed to fixate on a cross located in the center of the screen at all times. Two colors,

16 red and blue, were used as cues for the participants. Whenever the cross was red, participants

17 were instructed to reduce their blinking comfortably (if possible), while still prioritizing the

18 listening task. Whenever the cross was blue, they were instructed to blink freely. Each stimulus

19 was preceded by a baseline period (used during analysis to establish pupil size prior to input)

20 with $3000 \mathrm{~ms}$ of silence and a red cross. During the stimulus the cross remained red, and after the

21 stimulus there was a delay period with $3000 \mathrm{~ms}$ of silence and a (continued) red cross. After the

22 recording window, the color of the cross changed to blue, and participants were instructed to

23 repeat what they heard aloud. An audio recorder was used to record their responses. After the 


\section{PUPIL RESPONSE FOR ACCENTED SPEECH}

1 participant clicked the spacebar to initiate a new trial, there was a final $3000 \mathrm{~ms}$ silent delay with

2 a blue cross to allow the pupil response to recover.

3 Sixty stimuli (30 per condition) were presented in a randomized order. ${ }^{1}$ For subjective

4 ratings of listening effort, participants were probed every three trials about the last sentence they

5 had listened to and used the keyboard to respond, resulting in approximately 10 responses per

6 condition. Participants responded using a scale of 1 to 9 where: $1=$ "not effortful", $5=$

7 "moderately effortful", and $9=$ "extremely effortful." All participants were debriefed after the

8 experiment.

9 III. ANALYSES

10 1. Subjective Responses

11 For each participant, there were approximately ten responses to the effort probes for each

12 speaker. Their mean was used as a composite rating score. Quantile-quantile plots of the scores

13 demonstrated that they were not normally distributed, so Wilcoxon rank-sum tests were used to

14 compare ratings for the two speakers.

\section{2. Pupil Data Maintenance}

16 Recognition accuracy was scored by a single researcher, and trials in which any keywords were

17 missed were excluded from the pupillometry analyses (2.1\% of total trials). A custom script

18 written in $\mathrm{R}$ was used to manage pupil data. ${ }^{2}$ This maintenance pipeline began by aligning data

19 from each trial at sentence onset, and then averaging the pupil diameter during the 500ms

20 preceding each target sentence to determine a baseline value for each trial. Timepoints with

\footnotetext{
${ }^{1}$ A pilot experiment with a blocked design is reported in the Supplemental Materials. The main effect of accent is present in both designs.

${ }^{2}$ All pupil diameter measures are reported in the unit of measurement automatically provided by the EyeLink 1000 Plus system, called "arbitrary units."
} 


\section{PUPIL RESPONSE FOR ACCENTED SPEECH}

1 missing data due to blinks were omitted when calculating the baseline value, and trials with

2 fewer than $50 \%$ valid points during this window were omitted. Next, these baseline values were

3 subtracted from pupil diameter throughout the rest of each trial to create a measure of absolute

4 change. To remove effects of blinking, intervals of missing values were identified and then

5 expanded to remove noisy readings caused by the eyelids moving prior to (by $30 \mathrm{~ms}$ ) and

6 following (by 160ms) blinks (Winn et al., 2015; Zekveld et al. 2010). This expanded blink

7 interval was then interpolated across using linear approximation. Lastly, the pupil data was

8 smoothed using a 10Hz moving average (Winn, Wendt, Koelewijn, \& Kuchinsky, 2018). Trials

9 that required more than 50\% interpolation were excluded from analyses. Across all participants

10 and trials, $2.9 \%$ of timepoints were identified as blinks and interpolated. Across participants, less

11 than one trial was excluded on average for this reason, and no participant exceeded $20 \%$ trial

12 loss.

\section{4. Growth Curve Analysis}

14 Growth curve analysis (GCA) was used to model the pupil data in R (version 3.5.1) using the

15 lme4 package. GCA is similar to polynomial regression, but controls for potential collinearity

16 issues by orthogonalizing the polynomial time terms (Mirman, 2014). For the present study, we

17 opted to use GCA instead of more traditional mean pupil diameter and peak pupil diameter

18 measures for the following reasons: 1) GCA allows for analysis of the full shape of the pupil

19 response, and analysis of all data in the time-course without averaging across trials (as is

20 necessary with ANOVA); 2) calculation of the peak pupil diameter from pupil data averages

21 (i.e., averages across trials within a given subject) can introduce noise into the analysis process;

22 and 3) tests of mean pupil diameter are still present in GCA models (for our model, the main

23 effect of condition), and peak pupil diameter can be indirectly tested by examining the slope and 


\section{PUPIL RESPONSE FOR ACCENTED SPEECH}

1 shape of a given GCA model. The window of data used for model fitting began at 500ms after

2 the stimuli began (based on visual inspection of the data) and ended 1000ms after the average

3 sentence offset time $(3344 \mathrm{~ms})$. The pupil data was time-binned, reducing the sampling rate from

$4500 \mathrm{~Hz}$ to $50 \mathrm{~Hz}$. Table I shows the full model and its specifications. The time-course of pupillary

5 response was modeled up to the third-order (cubic) orthogonal polynomial, based on hierarchical

6 model comparisons that indicated significant contributions of the quadratic $\left(\chi^{2}{ }_{1}=61.19 ; p<\right.$

$7 \quad 0.001)$ and cubic $\left(\chi^{2}{ }_{1}=5.84 ; p=0.016\right)$ polynomials to model fit. For the speaker condition

8 manipulation, dummy coding specified the native speaker as the reference (i.e., zero) group. The

9 random effects structure included random intercepts and slopes for subject and item. Correlations

10 between these slopes and intercepts were included in the random effects (as recommended by

11 Mirman, 2014).

\section{IV. RESULTS}

\section{1. Pupillometry}

14 The summary output from the full model is reported in Table I, and the model fit is shown in

15 Figure 1B. We predicted that there would be greater overall pupil response and a steeper increase

16 of pupil response for the L2 speaker condition than the native speaker condition, resulting in a

17 higher peak pupil response. The results confirmed both of these predictions, with a significant

18 main effect of condition $(\beta=59.40, S E=10.75, p<0.001)$ and a significant interaction between

19 the linear polynomial term and condition $(\beta=1061.73, S E=121.17, p<0.001)$. The interactions

20 between condition and the quadratic and cubic terms were not significant $(\beta=-14.24, S E=$

$2186.48, p=0.870$; and, $\beta=51.40, S E=47.37, p=0.282$, respectively), indicating that the shape

22 of the pupil response for each condition was similar. Overall, the pattern of results in the full 
1 model clearly demonstrate larger pupil response — and, thus, greater cognitive load — for unfamiliar L2-accented speech.
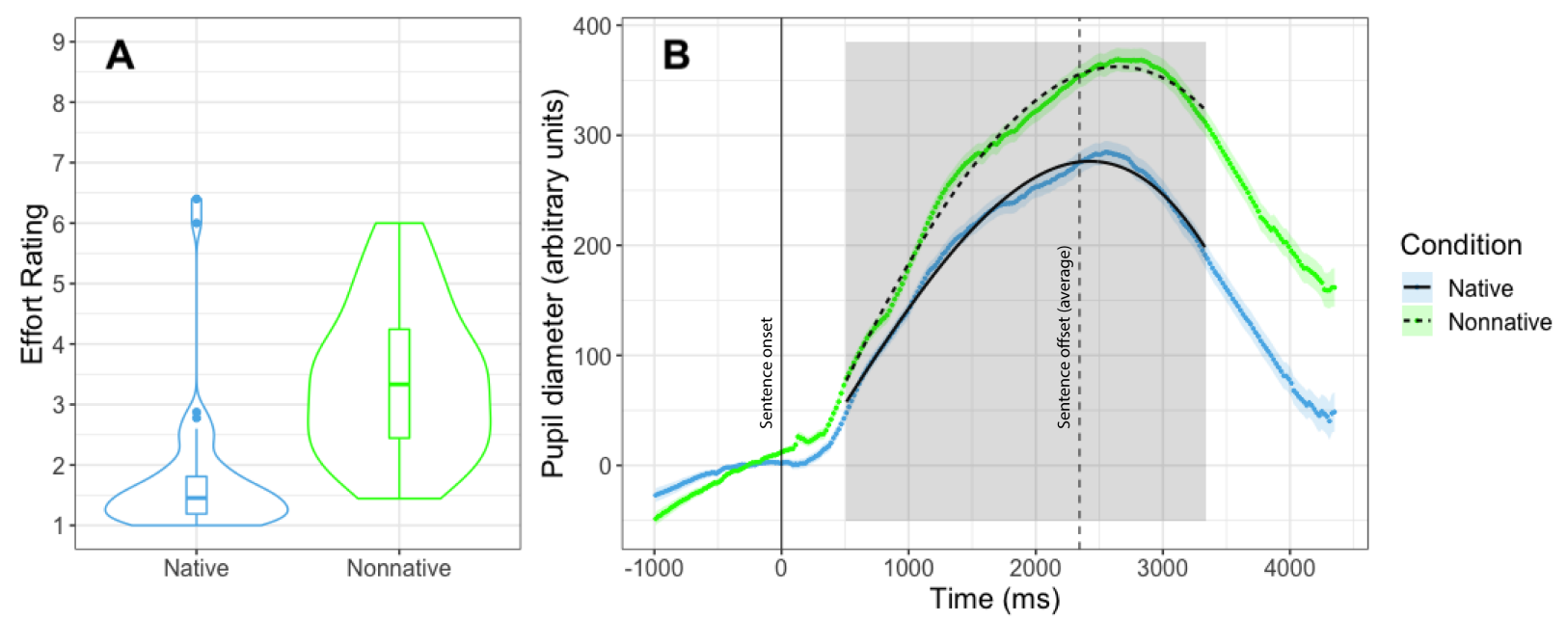

Fig. 1. A) Subjective ratings of listening effort are shown using a combination of box-andwhisker and violin plots. The response scale went from 1 to 9 , where: $1=$ "not effortful", $5=$ "moderately effortful", and 9 = "extremely effortful." B) Pupil diameter over time is plotted as a function of speaker. Points and ribbons are used to represent the raw data means and standard errors, and model fit is overlaid with black lines. Onset time of all sentences is represented as a solid vertical line, and the average sentence offset time (i.e., 2344ms) for both conditions is shown as a dashed vertical line. The gray box indicates the area of interest used for modeling the pupil response. Pupil diameter is measured in "arbitrary units" on the y-axis, which are the units automatically provided by the EyeLink 1000 Plus system.

\section{2. Subjective Responses}

15 As shown in Figure 1A, the L2 speaker was rated as more effortful to understand than the native 16 speaker $(p<0.001,95 \% \mathrm{CI}=[-2.03,-1.31])$.

\section{IV. DISCUSSION}

18 The results of the current study indicate that processing accurately-recognized, unfamiliar L2-

19 accented speech imposes greater cognitive load than native speech. These findings are consistent

20 with an executive recruitment account for accented speech, under which we would predict that

21 mismatches between the speech patterns of a speaker and a listener's representations will require

22 more cognitive resources to process - even when recognition accuracy is at ceiling. The 


\section{PUPIL RESPONSE FOR ACCENTED SPEECH}

1 pupillometry data thus provides a novel and more nuanced account of unfamiliar accented

2 speech processing, capturing differences in effort that standard measures of recognition accuracy

3 (i.e., "intelligibility") cannot. The one way in which recognition accuracy measures have been

4 used to study highly intelligible speech is by presenting the speech in noise (to bring

5 intelligibility off ceiling). Pupillometry has the distinct advantage of not requiring acoustic signal

6 degradation. However, the limitations of the study design should also be noted. Most

7 importantly, the current study uses a single talker in each condition, so it will be important to

8 verify that these results can be replicated with other types of unfamiliar accented speech. Future

9 work could expand upon these results by measuring pupil response for multiple speakers of a

10 given accent, and for multiple types of unfamiliar nonnative accents and regional dialects.

11 Our findings also indicate that listeners are aware that processing unfamiliar L2-accented

12 speech is more cognitively demanding than processing native speech. Complementing the

13 pupillometric findings, participants also rated the highly proficient L2 speaker as significantly

14 more effortful to understand than the native speaker (see also Munro \& Derwing, 1995).

15 However, while these subjective ratings match the physiological evidence, it is also possible that

16 they reflect participants' expectations and/or biases regarding accented speech, and not their

17 awareness of increased cognitive load. That is, participants may have rated the unfamiliar L2-

18 accented trials as more effortful because accented speech is stereotypically more difficult to

19 understand than native speech.

20 Investigating the executive function(s) underlying the increased cognitive load for

21 unfamiliar L2-accented speech will also be an important direction for future research. Current

22 speech perception models, such as the Ease of Language Understanding model (ELU; Rönnberg,

23 Rudner, Foo, \& Lunner, 2008; Rönnberg, et al., 2013), propose a dual role of working memory 


\section{PUPIL RESPONSE FOR ACCENTED SPEECH}

1 during speech perception, emphasizing that it is important both for storage of the unfolding

2 speech stream and for top-down processing of phonetic and semantic ambiguity. In line with this

3 model, the current literature suggests that working memory may be particularly important for

4 resolving perceptual ambiguities in unfamiliar accented speech. Both McLaughlin, Baese-Berk,

5 Bent, Borrie, and Van Engen (2018) and Janse and Adank (2012) found that working memory

6 capacity is positively related to recognition accuracy for accented speech in young and older

7 adults, respectively. While the ELU model primarily focuses on acoustically degraded speech,

8 the current findings motivate exploration of how individual differences in working memory may

9 play a critical role in unfamiliar accent processing, and, possibly, be reflected in pupil response

10 during listening.

11 In summary, our findings indicate that processing unfamiliar L2-accented speech imposes

12 greater cognitive load than processing native speech, as evidenced by a larger and more rapid

13 pupil response. Most notably, recognition accuracy for the unfamiliar L2-accented speech stimuli

14 used in the current study was at ceiling, indicating that mismatches between the speech patterns

15 of L2-accented speech and native listeners' representations require greater support from

16 executive resources (such as working memory) to reconcile. Additionally, while the accented

17 stimuli in the present study were recordings of a Mandarin-Chinese accented speaker of

18 American English, we would predict these findings to replicate with other unfamiliar L2 and

19 regional accents as well. If - as we have proposed - increased cognitive load stems from the

20 mismatches between the speech patterns of a speaker and a listener's linguistic representations,

21 then there should be greater pupil response for other unfamiliar accent types as well. Testing the

22 boundaries of this prediction will be an important next step for this area of inquiry. 


\section{PUPIL RESPONSE FOR ACCENTED SPEECH}

Acknowledgements

3 Fellowship awarded to Drew J. McLaughlin (DGE-1745038).

4

5

6 
PUPIL RESPONSE FOR ACCENTED SPEECH

\section{References}

2 Adank, P., Evans, B. G., Stuart-Smith, J., and Scott, S. K. (2009). “Comprehension of familiar and unfamiliar native accents under adverse listening conditions," J. of Exp. Psychol.:

$4 \quad$ Human Percept. and Perform., 35(2), 520.

5 Beatty, J. (1982). “Task-evoked pupillary responses, processing load, and the structure of processing resources,” Psychol. Bul. 91(2), 276.

7 Beatty, J., and Lucero-Wagoner, B. (2000). “The pupillary system,” Handbook Psychophys. 2, $142-162$.

9 Boersma, P. and Weenink, D. (2019). Praat: doing phonetics by computer [Computer program]. Version 6.0.50, Retrieved 31 March 2019 from http://www.praat.org/.

11 Francis, A. L., Tigchelaar, L. J., Zhang, R., and Zekveld, A. A. (2018). "Effects of second

Janse, E., and Adank, P. (2012). "Predicting foreign-accent adaptation in older adults," Q. J. of

16 McLaughlin, D. J., Baese-Berk, M. M., Bent, T., Borrie, S. A., and Van Engen, K. J. (2018). “Coping with adversity: Individual differences in the perception of noisy and accented

19 Mirman, D. (2014). Growth curve analysis and visualization using R. Boca Raton, FL: CRC Press.

21 Munro, M. J., and Derwing, T. M. (1995). "Foreign accent, comprehensibility, and intelligibility in the speech of second language learners," Lang. Learning 45(1), 73-97. 


\section{PUPIL RESPONSE FOR ACCENTED SPEECH}

1 Peysakhovich, V., Vachon, F., and Dehais, F. (2017). "The impact of luminance on tonic and phasic pupillary responses to sustained cognitive load,” Int. J. of Psychophys. 112, 40-45.

3 Pichora-Fuller, M. K., Kramer, S. E., Eckert, M. A., Edwards, B., Hornsby, B. W., Humes, L. E., ... and Naylor, G. (2016). "Hearing impairment and cognitive energy: The framework for understanding effortful listening (FUEL)," Ear Hear. 37, 5S-27S.

Porretta, V., and Tucker, B. V. (2019). "Eyes wide open: Pupillary response to a foreign accent varying in intelligibility," Front. in Comm. 4, 8.

Rönnberg, J., Lunner, T., Zekveld, A., Sörqvist, P., Danielsson, H., Lyxell, B., ... and Rudner, M. (2013). "The Ease of Language Understanding (ELU) model: theoretical, empirical, and clinical advances," Front. in Syst. Neurosci. 7, 31.

Rönnberg, J., Rudner, M., Foo, C., and Lunner, T. (2008). “Cognition counts: A working memory system for ease of language understanding (ELU),” Int. J. of Audiol. 47 (sup2), S99-S105.

Steinhauer, S. R., Siegle, G. J., Condray, R., and Pless, M. (2004). "Sympathetic and parasympathetic innervation of pupillary dilation during sustained processing," Int. J. of Psychophys. 52(1), 77-86.

Van Engen, K. J., and McLaughlin, D. J. (2018). "Eyes and ears: Using eye tracking and pupillometry to understand challenges to speech recognition,” Hear. Res. 369, 56-66.

Van Engen, K. J., and Peelle, J. E. (2014). "Listening effort and accented speech,” Front. in Human Neurosci. 8, 577.

Van Engen, K. J., Chandrasekaran, B., and Smiljanic, R. (2012). "Effects of speech clarity on recognition memory for spoken sentences," PLoS One, 7(9), e43753. 


\section{PUPIL RESPONSE FOR ACCENTED SPEECH}

1 Wang, H., and van Heuven, V. J. (2006). “Acoustical analysis of English vowels produced by

2 Chinese, Dutch and American speakers," Ling in the Netherlands, 237-248.

3 Winn, M. B., Edwards, J. R., and Litovsky, R. Y. (2015). "The impact of auditory spectral

$4 \quad$ resolution on listening effort revealed by pupil dilation," Ear Hear. 36(4), e153.

5 Winn, M. B., Wendt, D., Koelewijn, T., and Kuchinsky, S. E. (2018). "Best practices and advice

6 for using pupillometry to measure listening effort: An introduction for those who want to

7 get started," Trends in Hear. 22, 2331216518800869.

8 Zekveld, A. A., Kramer, S. E., and Festen, J. M. (2010). "Pupil response as an indication of

9 effortful listening: The influence of sentence intelligibility," Ear Hear. 31(4), 480-490.

10 Zekveld, A. A., and Kramer, S. E. (2014). "Cognitive processing load across a wide range of

11 listening conditions: Insights from pupillometry,” Psychophys. 51, 277-284. 


\section{PUPIL RESPONSE FOR ACCENTED SPEECH}

1 Tables

2 TABLE I

3

4 Growth curve analysis model code and summary output. "ot1", "ot2", and "ot3" refer to the

5 linear, quadratic, and cubic polynomial time terms, respectively. Data was dummy coded such

6 that the native speaker condition was the "Intercept" term.

\begin{tabular}{|c|c|c|c|c|c|}
\hline \multicolumn{6}{|l|}{ R Code: } \\
\hline \multirow{2}{*}{$\begin{array}{l}\text { lmer(Diameter } \\
\text { Term }\end{array}$} & \multicolumn{3}{|c|}{$\begin{array}{l}(\text { ot } 1+\text { ot } 2+\text { ot } 3) * \text { Condition }+ \\
(1+\text { ot } 1+\text { ot } 2+\text { ot } 3 \mid \text { Subject })+ \\
(1+\text { ot } 1+\text { ot } 2+\text { ot } 3 \mid \text { Item }) \\
\text { data }=\text { PRA_E2R_TB50_AOI.df })\end{array}$} & \multicolumn{2}{|c|}{$\begin{array}{l}\text { \# fixed effects } \\
\text { \# random effects } \\
\text { \# random effects }\end{array}$} \\
\hline & Estimate & SE & DF & $t$ & $p$ \\
\hline Intercept (Native) & 180.73 & 16.30 & 77.31 & 11.085 & $<0.001 * * *$ \\
\hline ot1 & 1061.73 & 121.17 & 77.89 & 8.763 & $<0.001 * * *$ \\
\hline ot2 & -596.76 & 87.84 & 102.36 & -6.793 & $<0.001 * * *$ \\
\hline ot3 & -118.10 & 44.58 & 96.16 & -2.649 & $0.009 * *$ \\
\hline Condition (Nonnative) & 59.85 & 10.75 & 58.14 & 5.565 & $<0.001 * * *$ \\
\hline ot1:Condition (Nonnative) & 453.50 & 83.53 & 58.02 & 5.429 & $<0.001 * * *$ \\
\hline ot 2:Condition (Nonnative) & -14.24 & 86.48 & 58.41 & -0.165 & 0.870 \\
\hline ot3:Condition (Nonnative) & 51.40 & 47.37 & 58.08 & 1.085 & 0.282 \\
\hline
\end{tabular}

7 
PUPIL RESPONSE FOR ACCENTED SPEECH

Supplemental Materials

\section{I. PILOT EXPERIMENT}

\section{A. Overview}

4 In the main experiment, we controlled for systematic differences in the speaking rates of the

5 native English speaker and the Mandarin-accented speaker by lengthening the native stimuli.

6 Here, we report the results of a pilot experiment in which we presented the stimuli without any

7 manipulation. The predictions were the same as the experiment reported in the main text: the

8 overall mean pupil diameter and the rate of change of pupil diameter would be greater for the

9 L2-accented speaker than the native-accented speaker, and the L2-accented speaker would be

10 subjectively rated as more effortful to understand than the native speaker. An additional rating

11 measure was included to assess how accented the speakers were each perceived to be as a

12 manipulation check to confirm that the L2 speaker was perceived as accented. All data files and

13 stimuli are available at: https://osf.io/7dajv/files/.

\section{B. Methods}

15 1. Participants

16 Young adult participants $(N=30,20$ female and 10 male, ages 18-27) were recruited from

17 Washington University's Psychology Subjects Pool. Eight additional participants were excluded

18 and replaced because of data loss and/or equipment malfunction, or reports that they were not

19 native speakers of American English, had parents who were not native speakers of American

20 English, or had extensive exposure to Mandarin Chinese. Pure tone audiometry was administered

21 for each participant to confirm that they had normal hearing. Participants were compensated with

22 class credit.

23 2. Stimuli 


\section{PUPIL RESPONSE FOR ACCENTED SPEECH}

1 Stimuli were the same as those used in the main experiment; however, for the pilot experiment

2 the recordings of the native speaker were not lengthened. Thus, the recordings for both speakers

3 were natural, and the Mandarin-accented stimuli were generally longer.

4 3. Procedure

5 The procedure matched the main experiment with the following exceptions: stimuli were blocked

6 by speaker, such that the main section was comprised of four blocks that alternated between

7 speaker type (i.e., 15 stimuli each). Blocking was counterbalanced across subjects. After each

8 block, participants were asked to rate the speaker on a scale of 1 to 9 for how effortful they were

9 to understand (where $1=$ "not effortful", 5 = "moderately effortful", and 9 = "extremely

10 effortful") and how accented they sounded (where $1=$ "native English speaker", 5 = "moderately

11 accented", and 9= "extremely accented"). Verbal responses were recorded by a researcher in the

12 room and a recording device. The timing of the trial-by-trial design also differed slightly: each

13 stimulus was preceded by a $2000 \mathrm{~ms}$ quiet baseline period and followed by a $2000 \mathrm{~ms}$ delay

14 period, during which the cross was red. After the red recording period, the color of the cross

15 changed to green, cuing the participant to repeat what they heard aloud. Colors were changed to

16 red and blue in the main experiment to accommodate participants with color blindness.

\section{C. Analysis}

18 The same custom R script was used to manage pupil data for the main experiment and the pilot

19 experiment. The only difference when processing the pilot data was that the pipeline began by

20 aligning data from each trial at sentence offset, with the intent of aligning the peak responses

21 across trials regardless of stimuli length (Klingner, Tversky, \& Hanrahan, 2011). Across all

22 participants and trials, $2.4 \%$ of timepoints were identified as blinks and interpolated across. The 
1 window for analysis began at average onset time of the accented trials $(-2327 \mathrm{~ms})$ and ended

$2 \quad 1000 \mathrm{~ms}$ after sentence offset time.

\section{D. Results}

\section{$4 \quad$ 1. Pupil Data}

5 Supplemental Table II contains output from the full model, and Supplemental Figure 2 shows the

6 raw data with the model fit overlaid. The largest random effect structure that would converge included random

7 intercepts and slopes for the subject-by-condition term on each of the orthogonal polynomial

8 terms. This random effect structure captures individual differences in the effect of the condition

9 manipulation on each subject (p.68, Mirman, 2014), and, thus, should capture more variance than

10 the subject random effect would alone. In comparing models, we found the model reported in

11 Table I to be the most conservative.

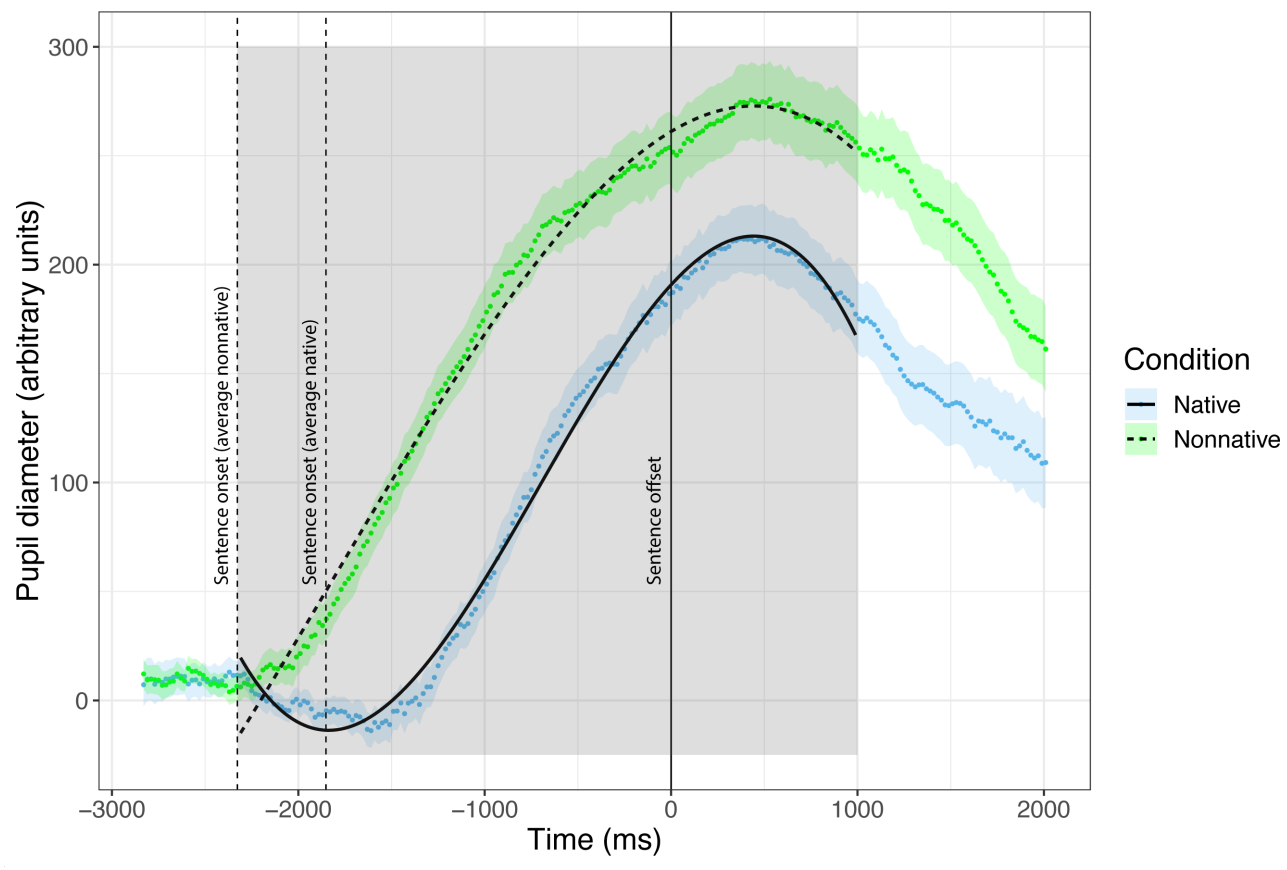

13 Supp. Fig. 2. Data from the pilot experiment show larger pupil response for L2-accented than 14 native speech. Raw data means and standard errors are represented as points and ribbons, and 15 model fit is overlaid with black lines. The solid vertical line represents the aligned offset time for all sentences, and the two dashed vertical lines represent average sentence onset times for the native (rightmost) and L2-accented (leftmost) conditions. The gray box indicates the selected 


\section{PUPIL RESPONSE FOR ACCENTED SPEECH}

1 area of interest used for modeling the pupil response. Pupil diameter is measured in arbitrary

2 units on the y-axis.

As predicted, there was a significant effect of condition $(\beta=75.51, S E=29.62, p=$

5 0.013), reflecting greater mean pupil diameter for the L2 speaker condition than the native

6 speaker condition. The interactions between condition and the linear and cubic terms were also

$7 \quad$ significant $(\beta=1,109.12, S E=351.68, p=0.003$; and, $\beta=982.81, S E=205.09, p<0.001$,

8 respectively). The interaction with the linear term - while consistent with our hypotheses - is

9 likely attributable to the systematic difference in stimuli length between the two conditions (i.e.,

10 the L2 speech was slower), and not due to a difference in the rate at which pupil diameter

11 increased. Additionally, the interaction between condition and the cubic term appears to be

12 present due to the alignment of the two conditions, and not for theoretically notable reasons (i.e.,

13 two inflection points of the native curve, and only one of the L2 curve, are captured in the

14 analysis window; Supplemental Figure 2). Thus, the systematic difference in stimuli length

15 complicated the interpretation of the results and motivated the editing of the native speaker files

16 for the main experiment.

\section{2. Subjective Responses}

18 Results of Wilcoxon rank-sum tests found that the L2 speaker was rated as more effortful to

19 understand than the native speaker $(p<0.001,95 \% \mathrm{CI}=[-3.00,-2.00])$, and as sounding more

20 accented $(p<0.001,95 \% \mathrm{CI}=[-4.50,-3.75])$.

\section{E. Brief Conclusion}

22 Moving forward, we recommend that researchers investigating speaker differences using

23 pupillometry carefully control for stimuli length between conditions. While the primary result of

24 the present study remained the same between the pilot and the main experiment (i.e., the 


\section{PUPIL RESPONSE FOR ACCENTED SPEECH}

1 differences in mean and peak pupil diameter), the interpretation of the shape of the pupil curve

2 was largely affected. 
PUPIL RESPONSE FOR ACCENTED SPEECH

1

2

\section{3}

6 such that the native speaker condition was the "Intercept" term.

Tables

SUPPLEMENTAL TABLE I

4 Growth curve model code and summary output from Experiment 1. "ot1", "ot2", and "ot3" refer

5 to the linear, quadratic, and cubic polynomial time terms, respectively. Data was dummy coded

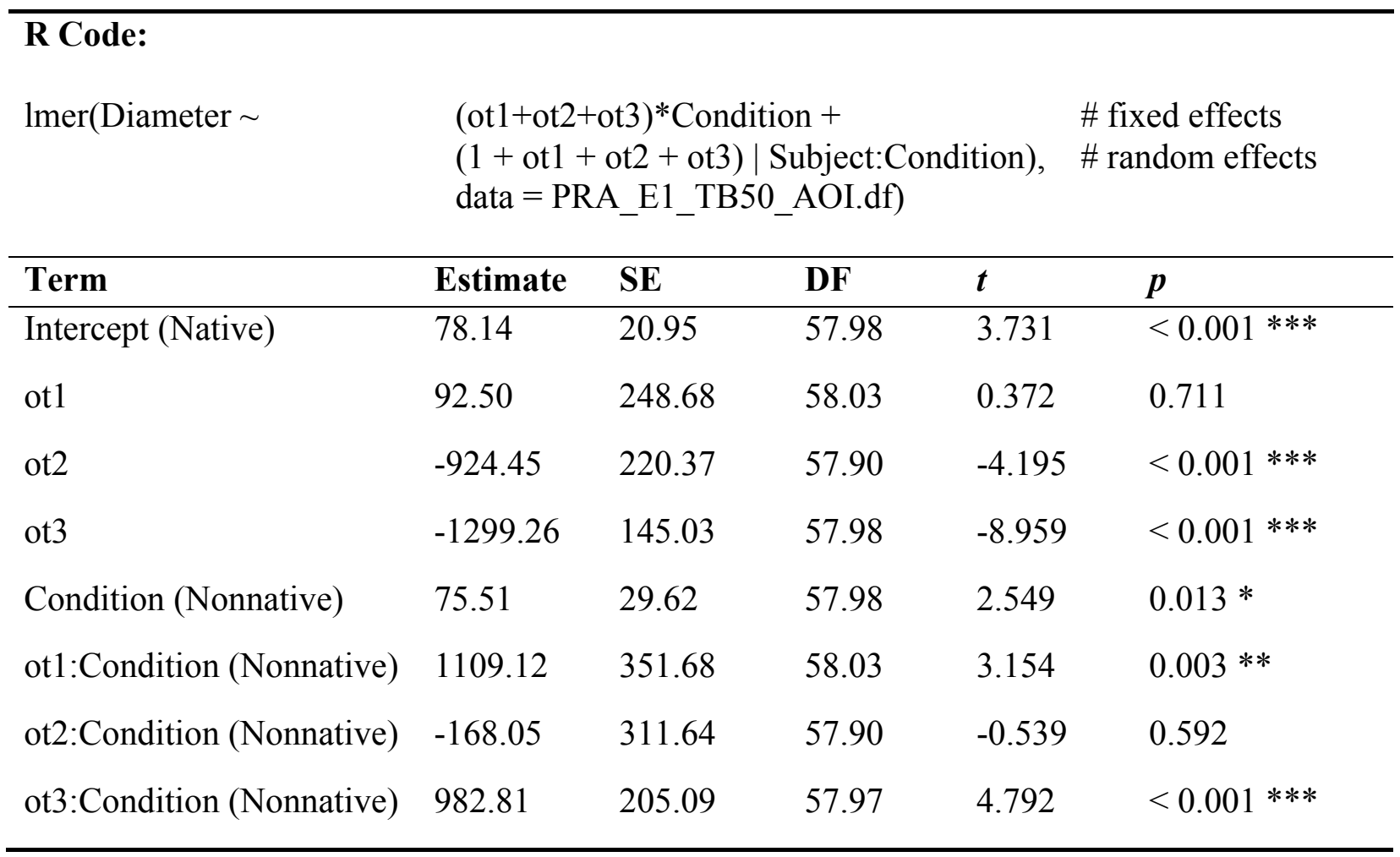

